\title{
Contribution of maternal effect QTL to genetic architecture of early growth in mice
}

\author{
JB Wolf, TT Vaughn, LS Pletscher and JM Cheverud \\ ${ }^{1}$ Department of Ecology and Evolutionary Biology, University of Tennessee, Knoxville, TN 37996-1610, USA; ${ }^{2}$ Department of \\ Anatomy and Neurobiology, Washington University School of Medicine, St. Louis, MO 63110, USA
}

\begin{abstract}
Existing approaches to characterizing quantitative trait loci (QTL) utilize a paradigm explicitly focused on the direct effects of genes, where phenotypic variation among individuals is mapped onto genetic variation of those individuals. For many characters, however, the genotype of the mother via its maternal effect accounts for a considerable portion of the genetically based variation in progeny phenotypes. Thus the focus on direct effect QTL may result in an insufficient or misleading characterization of genetic architecture due to the omission of the potentially important source of genetic variance contributed by maternal effects. We analyze the relative contribution of direct and maternal effect (ME) QTL to early growth in mice using a three-generation intercross of the Small (SM/J) and Large (LG/J) inbred mouse lineages. Using interval mapping and composite interval mapping, direct effect (DE) QTL for early growth (change in body mass during the interval from week 1 to 2 ) were detected in the $\mathrm{F}_{2}$ generation of the intercross $(n=510)$, where no maternal genetic effect variance is present (all individuals are progeny
\end{abstract}

of genetically identical $F_{1}$ mothers). ME QTL were detected by treating the phenotypes of cross-fostered $F_{3}$ pups as a characteristic of their nurse-dam ( $n=168$ dams with crossfostered progeny). Five DE QTL, significant at a chromosome wide level $(\alpha=0.05)$, were detected, with two significant at a genome wide level. FourME QTL significant at the chromosome wide level were detected, with three significant at the genome wide level. A model containing only DE QTL accounted for $11.8 \%$ of phenotypic variance, while a model containing only ME QTL accounted for $31.5 \%$ of the among litter variance in growth. There was no evidence for pleiotropy of DE and ME loci since there was no overlap between loci detected in these two analyses. Epistasis between all pairs of loci was analyzed for both DEs and MEs. Ten pairs of loci showed significant epistasis for MEs ( $\alpha=0.05$ corrected for multiple comparisons) while four pairs showed significant epistasis for DEs on early growth.

Heredity (2002) 89, 300-310. doi:10.1038/sj.hdy.6800140

Keywords: development; genetic architecture; mouse; maternal effects; quantitative trait loci

\section{Introduction}

Characterization of quantitative trait loci (QTL) contributing to genetic variation underlying complex phenotypes is becoming commonplace. Existing analyses have begun to elucidate the genetic architecture of quantitative traits, including factors such as number of loci, relative magnitude of their effects, degree of pleiotropy and linkage, presence of epistasis and distribution of loci across the genome (see Lynch and Walsh 1998 for a review of QTL analysis). QTL are usually identified by looking for correlations between the allelic state at chromosomal regions and the phenotypic value of traits of individuals. When these associations exist these chromosomal regions represent QTL. Implicit in this approach is the assumption that the genes possessed by an individual have direct effects on that individual's phenotype (ie, there is a direct mapping from the genotype to the phenotype). These effects are known as direct genetic effects to reflect this direct mapping (see Wolf et al, 1998).

Correspondence: JB Wolf, Department of Ecology and Evolutionary Biology, 569 Dabney Hall, University of Tennessee, Knoxville, TN 379961610, USA. E-mail: jbwolf@utk.edu

Received 1 November 2001; accepted 29 May 2002
While the assumption of direct mapping may be met for many characters, and has allowed for the characterization of loci contributing to a variety of traits, there are important instances where this requirement is not met. Indirect genetic effects can occur, where the genotype of one individual influences the expression of a phenotype of another individual (see Wolf et al, 1998). The presence of indirect genetic effects means that the genotype of an individual maps to the phenotype of a different individual, and as a result, these effects are not detected in the traditional direct mapping approach of QTL analysis. The most notable instance of indirect genetic effects occurs when the genotype of the mother influences the phenotype of her offspring beyond her direct contribution of DNA (reviewed by Mousseau and Fox, 1998a). These socalled maternal genetic effects can account for much of the phenotypic variance of early developmental characters in many taxa (eg, mice (Falconer, 1965), fish (Reznick, 1991), plants (Roach and Wulff, 1987), and insects (Mousseau and Dingle, 1991)) and occasionally contribute significant variation in traits expressed later in life (Mousseau and Fox, 1998b). In fact, the contribution of maternal effects to variation in many early developmental characters can be greater than the contribution of the individual's own genotype (eg, Oksh et al, 1967). 
While maternal effect loci have generally not been included in genetic analyses there is no reason to believe that they are any less important to the genetic architecture of complex phenotypes than are direct effect loci. They can contribute to the genetically based variation in a population that may underlie phenotypes of interest in applied mapping studies (such as pathologies [eg, Caldji et al, 2000; Reifsnyder et al, 2000]). Maternal genetic effects also provide heritable variation that can contribute to phenotypic evolution, and their influence on evolutionary processes can be very different from that of direct effects (see Cheverud and Moore 1994; Wolf et al, 1998). They can lead to time lags and momentum in the response to selection and can complicate multivariate evolution by modifying the genotype-phenotype relationship (Falconer, 1965; Kirkpatrick and Lande, 1989). Maternal effects are particularly important in the evolution of characters expressed early when variance for fitness (ie, opportunity for selection) is often large and maternal influences on expression of characters is most pronounced. In haplo-diploids, and possibly when Xlinked, maternal effect genes can also facilitate the evolution of maternal care and of genes with antagonistic pleiotropic effects on sons (Wade, 2001).

In mammals, where the maternal offspring association is particularly intimate and prolonged, maternal effects often account for as much as $50 \%$ of the variation in offspring traits, and at many ages, may even contribute effects on the same or greater order of importance as the offspring's own genotype (Cheverud and Moore, 1994). The quantitative genetic studies on characters expressed early in life in mammals clearly demonstrate that maternal effects are important components of genetic architecture and must be included in genetic analyses if one wishes to achieve a complete mechanistic understanding of phenotypic variation, where all sources of genetic variation are identified (eg, Reifsnyder et al, 2000). Inclusion of maternal effects in QTL analysis is also important if one wishes to attribute genetic effects to their correct source. Problems can arise because there is necessarily a correlation between an individual's genotype and the maternal genetic effect of their mother owing to Mendelian inheritance. This can make maternal effect loci appear to be direct effect loci and as a result, the lack of inclusion of maternal effects in QTL analyses may result in the incorrect characterization of maternal effect loci as direct effect loci (Wolf, 2000).

Maternal effects are particularly well characterized in the mouse, where classic quantitative genetic studies have demonstrated the importance of maternal effects in the genetics of complex phenotypes such as early size and growth (eg, Chai, 1956; Oksh et al, 1967). While there have been many molecular quantitative genetic studies of loci affecting growth in mice (see Corva and Medrano, 2001) no existing studies have included the contribution of maternal effect loci, despite their obvious importance in the quantitative genetics of growth. Here we analyze the contribution of maternal effects to the genetics of early developmental traits in mice by characterizing QTL influencing early growth. We use a three-generation intercross of Large (LG/G) and Small (SM/J) inbred strains of mice that allows for the separation of direct and maternal effect QTL. Previous quantitative genetic analysis of this intercross detected significant post-natal maternal effect variance for many characters, including growth (Kramer et al, 1998). Cheverud and colleagues have previously used this cross to characterized QTL contributing to a number of traits, including early body size and growth rate (eg, Cheverud et al, 1996; Vaughn et al, 1999), but have not included maternal effect loci in these analyses.

\section{Materials and methods}

\section{Breeding, husbandry and phenotypes}

The inbred mouse strains Large $(\mathrm{LG} / \mathrm{J})$, selected for large body size at 60 days (Goodale, 1938) and Small (SM/J), selected for small body size at 60 days (MacArthur, 1944), were intercrossed to create the mapping population used in this study. These lines show a 6-8 standard deviation difference in size related traits (Kramer et al, 1998). Other details of these strains are given in Cheverud et al (1996) and Vaughn et al (1999).

The study population was initiated by crossing 10 $\mathrm{SM} / \mathrm{J}$ males to $10 \mathrm{LG} / \mathrm{J}$ females to produce an $\mathrm{F}_{1}$ hybrid population of 52 animals. The $\mathrm{F}_{1}$ individuals were randomly mated producing $510 \mathrm{~F}_{2}$ individuals. $\mathrm{F}_{2}$ animals were randomly mated yielding $1632 \mathrm{~F}_{3}$ animals in 200 independent full-sib families. Males were removed from the mating cage after females were gravid, thereby eliminating the possibility of postnatal paternal effects. Females giving birth on the same day had their progeny cross-fostered. Cross fostering was done pair-wise, where half of a litter from one female was exchanged for half of a litter from one other female. In all, progeny in 158 of the 200 families were cross-fostered. Individuals were weaned at 21 days and placed into single sex cages of five animals each. Other details of husbandry are given in Cheverud et al (1996) and Vaughn et al (1999).

All individuals were weighed weekly starting at 7 days of age up until 10 weeks of age. Weights were recorded to the nearest $0.1 \mathrm{~g}$ with a digital balance. In this analysis we focus on growth from day 7 to 14 (early growth) because this period of growth occurs during the time of rapid development under maternal care.

The growth rate of each individual was calculated from the age-specific weights by subtraction. Growth, as opposed to age specific weight, was chosen to minimize the contribution of pre-natal maternal effects to phenotypic variance. Pre-natal maternal effects may still influence postnatal growth (eg, Cowley et al, 1989; Atchley et al, 1991), but we have diminished their contribution to phenotypic variance by removing variance contributed by intrauterine growth (manifested as variance in birth weight) and growth during the first week (which may also show strong pre-natal maternal effects). Each dam that received cross-fostered pups was assigned a maternal performance score based on the mean phenotype of the cross-fostered pups she reared. These maternal performance scores are the characters used in the analysis of maternal effects QTL.

\section{Quantitative genetics}

The relative contribution of direct and maternal effects to phenotypic variation was characterized by quantitative genetic analysis. Variance in early growth was partitioned into four components (dam, nurse-dam, dam-bynurse-dam interaction and residual variance) using restricted maximum likelihood (REML) in the Mixed pro- 
cedure of SAS (SAS ${ }^{\circledR}$ Institute Inc, 1992) treating dam, nurse-dam and dam-by-nurse-dam interaction as random variables (the full ANOVA model used is given by Riska et al, 1985). Upper and lower confidence limits $(\alpha=0.05)$ on the estimated variance components were estimated using Wald Z-scores and normal quantiles (see SAS ${ }^{\circledR}$ Institute Inc, 1992 for details). The dam component $\left(V_{\text {dam }}\right)$ is an estimate of the covariance of full-sibs raised by different dams and has an expected value of:

$$
V_{d a m}=\frac{1}{2} V_{a}+\frac{1}{4} V_{d}+\frac{1}{4} V_{a x a}+\frac{1}{8} V_{a x d}+\frac{1}{16} V_{d x d}+V_{m(\text { pre })}
$$

where $V_{a}$ is the additive genetic variance, $V_{d}$ is the dominance genetic variance, $V_{a x a}$ is the additive-by-additive epistatic variance, $V_{a x d}\left(=V_{d x a}\right)$ is the additive-by-dominance epistatic variance, $V_{d x d}$ is the dominance-by-dominance epistatic variance and $V_{m \text { (pre) }}$ is the pre-natal maternal effect variance. Because this component contains unequal fractions of the various components of variance, it cannot be used as a direct estimate of any single variance component. However, it can be doubled as a means of estimating the lower limit of the total genetic variance, which can be used to estimate broad sense heritability. The doubled dam variance contains a full contribution of additive genetic variance, but still contain less than a full contribution of the non-additive genetic effects. The doubled estimate also contains a double contribution of pre-natal maternal effect variance. However, this variance component is likely to be small given that the estimate of growth is calculated by subtracting off earlier size periods, and thus may not contain much of a contribution of prenatal effects.

The nurse-dam component $\left(V_{\text {nurse }}\right)$ is an estimate of the covariance of unrelated individuals raised by the same dam. This component estimates $V_{m \text { (post) }}$, the postnatal maternal effect variance, which includes both genetic and non-genetic sources of maternal effects (Riska et al, 1985). The dam-by-nurse-dam interaction term is an estimate of the interaction variance of the dam effect (direct genetic effects and the pre-natal maternal effect) with the nursedam effect (postnatal maternal effect). This term can be seen as an estimate of half the genotype-by-maternalenvironment interaction (Legates, 1972; Riska et al, 1985).

The genetic covariance between direct and maternal effects $\left[\operatorname{cov}\left(A_{o}, A_{m}\right)\right]$ was estimated following the approach of Riska et al (1985); see also Newman et al (1989). This approach relies on the fact that the expected covariance of full sibs raised by their own mother contains the direct-maternal genetic covariance, while the covariance of full sibs nursed by an unrelated dam does not. In this approach, each dam-nurse pair is used as an independent ( 1 d.f.) estimate of the direct-maternal covariance, which is calculated by subtracting the among-litter component for fostered pups from that for unfostered pups. The mean of these estimates is presented along with confidence limits calculated using the standard error of this estimate. Only dam-nurse pairs that had at least two individuals per combination were included in this analysis. In all, 69 dam-nurse pairs were included.

Because the breeding design does not allow for an estimate of the genetic variance of the maternal effect, the covariance between direct and maternal effects cannot be expressed as a genetic correlation. However, it is possible to establish a minimal value for the genetic correlation
$\left(r_{A O A M}\right)$ by assuming that the maternal effect is entirely genetically determined. Under this assumption, the genetic correlation can be calculated as:

$$
r_{A_{O} A_{M}}=\frac{\operatorname{cov}\left(A_{o}, A_{m}\right)}{\sqrt{V_{\text {nurse }} 2 V_{\text {dam }}}}
$$

Note that the dam variance component is doubled to represent the total genetic variance.

\section{Genotyping and QTL analysis}

Initially, 96 microsatellite loci arranged in 72 intervals covering all 19 autosomes were scored on the $F_{2}$ animals. The markers and their locations are listed in Table 1. Map distances for autosomal markers were calculated using the entire $\mathrm{F}_{2}$ population. Details of the autosomal map construction and other details of molecular genotype scoring are given in Vaughn et al (1999, see intercross II).

After the initial analysis of the autosomal loci an additional six loci on the $X$ chromosome were scored. Due to the fact that the distribution of genotypes on the $X$ chromosome and on the autosomes are so different (females have backcross genotypes while males are haploid) the analysis of the X-lined loci required different methods than the autosomal analysis. Since no loci were detected on the $X$ chromosome in any of the analyses, details of this analysis are not given here, and the rest of the methods focus on the autosomal analysis.

The regression interval mapping (IM) technique of Haley and Knott (1992) was used to impute genotype scores every $2 \mathrm{cM}$ in the intervals between molecular markers. The marker genotypes and levels of recombination were used to calculate the probability that positions between flanking markers were either homozygous $\mathrm{SM} / \mathrm{J}$, heterozygous or homozygous LG/J. The probabilities were multiplied by $-1,0$ and +1 respectively and then summed to obtain an additive genotypic score. Similarly, the dominance genotypic score was calculated by finding the probability that a location was heterozygous.

The presence and relative positions of potential QTL were determined by regressing phenotypic values onto the additive and dominance genotypic scores to obtain the probability of a gene affecting the character at the specified location. These probabilities were obtained by canonical correlation analysis (Blackith and Reyment, 1971) using SAS (Leamy et al, 1999). The most likely QTL position is the one with the lowest probability of having occurred by chance. The probability associated with the multiple regression at each QTL position was transformed to a linear scale by logarithmic transformation [LPR $=\log _{10}(1 /$ Prob.)] (see Cheverud et al, 2001) and, as a result, our LPR scores can be directly compared to LOD scores obtained through maximum likelihood methods (Lander and Botstein, 1989). Environmental covariates were included in the mapping to remove phenotypic variation. In the mapping of direct and maternal effects on early growth we included cohort and litter size at weaning as covariates. This covariate accounts for environmental variation introduced by the fact that not all individuals were born during the same period.

Confidence limits for these positions were determined by the interval encompassing a chromosomal region for which the increase in probability of a false positive result is less than an order of magnitude (Lander and Botstein, 1989). For chromosomes showing multiple valleys of 
Table 1 Microsatellite markers and distance from the most centromeric marker (Centro. Dist.) are given in Haldane's cM. The effective number of markers and the chromosome-wide significance threshold for each chromosome is given in parentheses after the chromosome name for $\alpha=0.05$

\begin{tabular}{|c|c|c|c|c|c|c|c|}
\hline Locus & $\begin{array}{c}\text { Centro. } \\
\text { Dist. }\end{array}$ & Locus & $\begin{array}{c}\text { Centro. } \\
\text { Dist. }\end{array}$ & Locus & $\begin{array}{c}\text { Centro. } \\
\text { Dist. }\end{array}$ & Locus & $\begin{array}{c}\text { Centro. } \\
\text { Dist. }\end{array}$ \\
\hline Chr. $1(6.6,2.13)$ & & Chr. $5(5.2,2.01)$ & & Chr.9 (3.4, 1.83) & & Chr.14 (3.5, 1.84) & \\
\hline D1Mit3 & 0 & D5Mit47 & 0 & D9Mit2 & 0 & D14Nds1 & 0 \\
\hline D1Mit20 & 10 & D5Mit61 & 20 & D9Mit4 & 14 & D14Mit5 & 44 \\
\hline D1Mit74 & 32 & D5Mit6 & 76 & D9Mit19 & 72 & D14Mit7 & 64 \\
\hline D1Mit7 & 44 & D5Mit26 & 86 & & & D14Mit266 & 88 \\
\hline D1Mit11 & 56 & D5Mit165 & 118 & Chr.10 (3.6, 1.90) & & & \\
\hline D1Mit14 & 78 & D5Mit43 & 130 & D10Mit2 & 0 & Chr.15 (3.2, 1.81) & \\
\hline D1Mit17 & 120 & & & D10Mit15 & 36 & D15Mit13 & 0 \\
\hline \multirow[t]{2}{*}{ D1Mit155 } & 136 & Chr. $6(4.3,1.93)$ & & D10Mit65 & 46 & D15Mit5 & 24 \\
\hline & & D6Mit1 & 0 & D10Mit10 & 68 & D15Mit2 & 50 \\
\hline Chr. 2 (5.2, 2.02) & & D6Mit9 & 50 & D10Mit133 & 76 & D15Mit42 & 76 \\
\hline D2Mit1 & 0 & $D 6 N d s 5$ & 68 & D10Mit14 & 88 & & \\
\hline D2Mit370 & 46 & D6Mit58 & 92 & & & Chr.16 $(1.7,1.53)$ & \\
\hline D2Mit380 & 68 & D6Mit15 & 98 & Chr.11 (4.9, 1.99) & & D16Mit2 & 0 \\
\hline D2Mit17 & 92 & & & D11Mit62 & 0 & D16Mit5 & 30 \\
\hline D2Mit28 & 98 & Chr.7 (6.7, 2.11) & & D11Mit64 & 46 & & \\
\hline D2Mit22 & 116 & D7Mit21 & 0 & D11Mit15 & 58 & Chr.17 (2.3, 1.66) & \\
\hline \multirow[t]{2}{*}{ D2Mit265 } & 144 & D7Mit26 & 32 & D11Mit14 & 82 & D17Mit46 & 0 \\
\hline & & $D 7 N d s 1$ & 44 & D11Mit333 & 98 & D17Mit16 & 10 \\
\hline Chr. $3(5.0,2.00)$ & & D7Mit148 & 54 & D11Mit48 & 114 & D17Mit39 & 42 \\
\hline D3Mit54 & 0 & D7Mit17 & 58 & & & & \\
\hline D3Mit22 & 44 & D7Mit9 & 72 & Chr.12 (4.7, 1.98) & & Chr.18 (2.8, 1.74) & \\
\hline D3Mit12 & 62 & D7Mit71 & 82 & D12Mit37 & 0 & D18Mit12 & 0 \\
\hline D3Mit49 & 68 & D7Mit46 & 98 & D12Mit2 & 20 & D18Mit17 & 4 \\
\hline D3Mit194 & 110 & $D 7 N d s 4$ & 106 & D12Mit5 & 42 & D18Mit51 & 26 \\
\hline \multirow[t]{2}{*}{ D3Mit32 } & 124 & & & D12Mit6 & 52 & D18Mit79 & 46 \\
\hline & & Chr.8 (3.3, 1.82) & & D12Nds2 & 72 & & \\
\hline Chr. $4(3.5,1.85)$ & & D8Mit293 & 0 & & & Chr.19 (2.8, 1.75) & \\
\hline D4Mit2 & 0 & D8Mit25 & 24 & Chr.13 (3.6, 1.85) & & D19Mit16 & 0 \\
\hline D4Mit163 & 16 & D8Mit89 & 76 & D13Mit1 & 0 & D19Mit2 & 50 \\
\hline D4Mit17 & 32 & D8Mit56 & 88 & D13Mit115 & 10 & D19Mit35 & 58 \\
\hline D4Mit45 & 46 & & & D13Mit9 & 62 & D19Mit137 & 62 \\
\hline D4Mit16 & 68 & & & D13Mit35 & 98 & & \\
\hline D4Mit13 & 90 & & & & & & \\
\hline
\end{tabular}

probability, composite interval mapping (CIM) was employed, where the influence of linked loci on the same chromosome was removed from the variance at the first locus in order to obtain an independent significance value for each locus (Zeng, 1993). CIM was not used for all chromosomes since, when only a single peak was found, the inclusion of linked loci as covariates would simply reduce power while adding no precision in mapping (ie, we did not add marker covariates when they were not needed to improve mapping). Significance values are presented for the best-fit model (CIM or IM).

Epistasis between pairs of loci on separate chromosomes was analyzed using a modified version of the regression approach described above. Individuals were assigned additive-by-additive $(a-x-a)$, additive-by-dominance $(a-x-d)$, dominance-by-additive $(d-x-a)$ and dominance-by-dominance $(d-x-d)$ genotypic scores for all twolocus pairs by multiplying the appropriate single locus additive or dominance genotypic values for the pair of loci being analyzed (see Cheverud and Routman, 1995; Routman and Cheverud, 1997). Epistatic pairs of loci were identified by regressing phenotypic values onto the four epistatic genotype scores. The single locus additive and dominance effects were statistically controlled for by including the additive and dominance genotypic scores as covariates. Loci that were detected in the regression analysis containing the four epistatic effects were analyzed using a full model, where the single locus additive and dominance effects of the interacting loci were included along with the four epistatic effects. Estimates of the additive and dominance effects of both loci, as well as the a- $x-a, a-x-d, d-x-a$ and $d-x-d$ interactions effects were used to calculate significance values for the epistatic interaction using the method of Cheverud and Routman (1995; see also Routman and Cheverud, 1997). This last step is required because variance due to epistasis appears statistically as part of the single locus additive and dominance effects in the regression analysis, even though it originates from the interaction of the loci.

Chromosome-wide and genome-wide significance levels were determined based on the number of independent markers on each chromosome (ie, the effective number of markers) (Cheverud, 2000, 2001; Vaughn et al, 1999). The effective number of markers is less than the actual number of markers scored because of linkage disequilibrium between markers on the same chromosome. The effective number of markers was determined using the variance of the eigenvalues of the intermarker correlation matrix. These methods are described in Cheverud (2000, 2001). The 5\% chromosome-wide significance levels were obtained by dividing 0.05 by the effective number of markers on that chromosome. For a discussion of 
the merits of using a chromosome wide significance level see Cheverud et al (2001). The genome-wide significance level was obtained by dividing 0.05 by the effective number of markers in the genome. Effective numbers of markers and chromosome wide significance levels (expressed as LPR scores) are listed in Table 1. Overall, the effective number of markers is 76.2, which makes the genome wide significance cutoff LPR value 3.183. Cheverud (2001) has demonstrated that this method for determining significance levels when analyzing multiple comparisons in QTL data gives significance levels that are similar to those produced by the use of re-sampling techniques (eg, Churchill and Doerge, 1994).

To determine the number of independent marker pairs in the epistasis analysis the effective number of markers on each pair of chromosomes included in the analysis were multiplied and then summed. Overall the analysis included 2725 independent pair-wise comparisons. This number of combinations was then used as the divisor in the calculation of genome-wide $5 \%$ and $10 \%$ significance levels for the epistasis analysis. Pairs of loci that show an LPR value greater than 4.736 in the epistasis analysis were considered significant interactions at the $5 \%$ level, while pairs with a LPR greater than 4.435 were considered significant the $10 \%$ level. We included pairs of loci that were significant at the $10 \%$ level in the epistasis analysis because there were significantly more pairs of loci detected than expected by chance, suggesting that many combinations that did not surpass the genome wide significance level were true interactions. Because of this overabundance of epistasis we decided to use the more liberal cutoff in order to avoid the omission of important epistatic combinations. We also included pairs of loci that showed a significant interaction for at least one of the four epistatic terms. Because we did four independent analyses on each two-locus pair we used a more conservative LPR value of 5.342 as the $5 \%$ cutoff and 5.038 as the $10 \%$ cutoff for these values.

The three-generation intercross of $\mathrm{SM} / \mathrm{J}$ and $\mathrm{LG} / \mathrm{J}$ allows for the separation of maternal and direct effect loci. Direct effects were detected by analyzing the association between genotypes of the $\mathrm{F}_{2}$ individuals and the phenotypes of those same individuals. Because all $\mathrm{F}_{1}$ dams were genetically identical there are no genetically based maternal effects contributing to variation in the $\mathrm{F}_{2}$ phenotypes and as a result, all loci detected are necessarily direct effect loci. Maternal effect loci were detected by analyzing the association between the genotype of a nurse-dam and the mean phenotype of the cross-fostered progeny that she reared. Loci detected in this analysis are necessarily maternal effects loci since the only reason that a nurse-dam's genotype would correlate with the mean phenotype of cross-fostered progeny is the presence of a genetically based maternal effect. Note that there is significantly more power to detect direct effect loci, and direct effect epistasis, since the analysis of direct effects is based on 510 individuals while the analysis of maternal effects is based on only 158 individuals. As a result, the difference between the results for these two types of effects should be interpreted in this light. There is a more detailed presentation of this point in the discussion section, where we discuss the implications of the different sample sizes for the interpretation of the results of this study.

In order to avoid pseudoreplication, the mean values of entire cross-fostered litters were used to map the maternal effects. As a result, all maternal effect QTL have variance component estimates that are measured on an among litter scale, while direct effects are measured on an among-individual scale. Because these two scales are not directly comparable it is necessary to transform the among litter variance to a scale comparable to the amongindividual variance. Among-litter variance can be transformed to an among-individual scale because the two have a simple expected relationship that is a function of group (family) size. Any covariance parameter that results in resemblance of individuals within groups (such as maternal effects) leads to an among group component of variance, and as a result, the relationship between among-group variance and within-group covariance can be approximated by the equation:

$$
V_{\text {among }}=\frac{1}{n} V_{P}+\frac{n-1}{n} \operatorname{cov}_{\text {within }}
$$

where $n$ is the number of sibs in a cross fostered group, $V_{\text {among }}$ is the among litter variance, $V_{P}$ is the total phenotypic variance measured on an among individual scale and $\operatorname{cov}_{\text {within, }}$ is the covariance among individuals within a group (eg, resemblance owing to maternal effects).

\section{Results}

\section{Quantitative genetics of growth}

Table 2 gives the estimates of the dam, nurse-dam and dam-by-nurse-dam interaction variance components for early growth. Presented in the table are also portions of variance explained by each of these components. The genetic covariance of direct and maternal effects is also given. Confidence limits on the genetic covariance span zero, though most of the region in the confidence interval is positive, indicating the likelihood of a small positive covariance. The estimated lower limit to the genetic correlation $\left(r_{A_{O} A_{M}}\right)$, calculated using equation (2) is equal to 0.179 . This small genetic correlation implies that there is likely to be little shared genetic basis (ie, pleiotropy) for the direct and maternal effects on early growth, though it is important to remember that this provides a lower limit for the actual genetic correlation.

The broad sense heritability estimate of 0.121 for early growth indicates that we expect to find direct effect QTL that explain a relatively small portion of the total phenotypic variance. Because of the possible contribution of dominance and epistasis, which are under represented in

Table 2 Quantitative genetics of early growth (weight gain from week 1 to 2 in grams). Covariance parameters are defined in the text. The $v a r / V_{P}$ is the covariance parameter divided by the total phenotypic variance and is used to show the portion of total variance accounted for by the variance component. $95 \%$ confidence limits are also presented

\begin{tabular}{lccc}
\hline Component & (Co)variance & var / Vp & 95\% CLs \\
\hline dam & 0.030 & 0.061 & 0.014 to 0.105 \\
nurse-dam & 0.163 & 0.330 & 0.113 to 0.256 \\
dam-x-nurse & 0.018 & 0.037 & 0.006 to 0.333 \\
residual variance & 0.283 & 0.572 & 0.260 to 0.309 \\
total phen. var (Vp) & 0.495 & - & - \\
cov(DGE, MGE) & 0.0177 & - & -0.027 to 0.062
\end{tabular}


this estimate, this is a lower limit to the portion of variance that may be explained by QTL. The nurse component of 0.330 indicates that maternal effects account for about one-third of all among individual variance in early growth. Because there is no way to estimate the portion of this variance that is attributable to maternal genetic effects (as opposed to maternal environmental effects), this number can be viewed as the upper limit of the portion of variance that maternal genetic QTL will explain. Using equation (3) and a mean number of four pups in each cross fostered group, maternal effects would be expected to explain $43.4 \%$ of the among litter variance in week 1 to 2 growth (which is approximately 0.282 ).

\section{QTL}

Single locus effects: Table 3 lists the direct effect QTL affecting early growth that were detected at the $\alpha=0.05$ chromosome wide level. There are a total of five QTL detected, with two significant at the genome wide level $(\alpha=0.05)$. Two of the QTL significant at the chromosome wide level appear on chromosome 2. Because of the presence of two QTL on a single chromosome mapping on chromosome 2 was done using composite interval mapping in order to eliminate the contribution of linkage disequilibrium to the estimates of the single locus values.

Most direct effect loci show additive effects, with a mix of positive and negative effects (ie, the LG/J and SM/J alleles can have either positive of negative effects on early growth depending on the locus). This mixture of effects was expected since there is little difference between the strains in early growth rates (see Kramer et al, 1998). The dominance effects that were detected are mostly positive, indicating that alleles promoting faster growth were partially dominant to those promoting slower growth. There is overdominance at the chromosome 12 QTL, indicating that the heterozygote grows faster than either homozygote, and partial dominance for the chromosome 5 QTL. Individual loci account for between 1.8 and $3.0 \%$ of the variance in early growth, and the five loci taken together account for $11.8 \%$ of all of the among individual variance in early growth. Note that this is in line with the estimated broad sense heritability of this character, indicating that we are likely to have detected a large proportion of the loci with large to moderate effects on the phenotype.

Four maternal effect QTL affecting early growth were identified, three of which were significant at the genome- wide level (see Table 4). Two loci were mapped using interval mapping and two were mapped by composite interval mapping because those chromosomes showed multiple LPR peaks. In all cases, the removal of linkage disequilibrium in the CIM analysis resulted in a single LPR peak, and as a result, no chromosomes showed more than a single significant QTL. Dominance effects predominated over additive effects, with a mix of positive and negative dominance, indicating that, for some loci, progeny of the heterozygous mothers showed larger weight gain than the midpoint between the homozygotes, while the opposite was true at other loci. The chromosome 6 locus is under-dominant (where the heterozygote showed lower pup growth than either homozygote) while the chromosome 1 and 13 loci show over-dominance (where the heterozygote showed higher pup growth than either homozygote). Only chromosome 7 shows a significant additive effect. The maternal effect loci accounted for between 5.21 and $9.53 \%$ of the among litter variance in early growth. A model containing all four maternal effect loci accounts for a total of $31.5 \%$ of the among litter variance in growth, while a reduced model containing only the loci significant at the genome wide level accounts for $26.5 \%$ of the variance. With a mean number of four pups per cross-fostered group we would expect the estimated $31.5 \%$ of among litter variance to account for $23.9 \%$ of the variance among individuals. Similarly, we would expect the 5.21 and 9.53 percents contributed by the largest and smallest effect loci to account for 3.96 and 7.24 percent of the among individual variance respectively.

No direct effect early growth locus maps to the same chromosome as a maternal effect locus. Thus, we have no evidence for pleiotropy.

Epistasis: A total of 219 pairs of loci showing epistasis for direct effects on early growth were detected (identified as being significant at an unadjusted $P<0.05$ ). Some 137.5 combinations were expected by chance, and thus we have a clear overabundance of epistasis. Of these, six two-locus combinations were significant by our criteria (Table 5). One of these combinations shows overall epistasis that is significant at the 0.05 level. Four combinations were significant at the 0.10 level but not at the 0.05 level, but two of these had a component that itself was significant at the 0.05 level. The other combination does not show overall significant epistasis, but show a significant $a-x-d$ component of epistasis (at the 0.05 prob-

Table 3 Direct effect QTL. For each QTL the most proximate centromeric marker is listed with the telomeric distance form the marker to the QTL in cM (Dist. M.), the distance from the most centromeric marker to the QTL (Dist. C.), the confidence interval (CI) for the QTL, the LPR associated with the location and the percent of total phenotypic variance that the QTL accounts for. Map identifies the method used to map the QTL (IM = interval mapping and CIM = composite interval mapping). The additive (a) and dominance (d) genotypic values are listed with the significant values in bold. LPR values significant at the genome wide level are indicated with $a^{* *}$ while ${ }^{*}$ indicates a locus significant at the chromosome level

\begin{tabular}{lcccccccc}
\hline Marker & Dist. M. & Dist. C. & CI & LPR & \% var. & Map & $a$ & $d$ \\
\hline D2Mit370 & 4 & 50 & 44 to 64 & $2.987^{*}$ & 2.49 & CIM & $-\mathbf{0 . 1 3 4}$ \\
D2Mit28 & 16 & 114 & 104 to 116 & $2.231^{*}$ & 1.88 & CIM & $-\mathbf{0 . 1 3 6}$ \\
D5M26 & 18 & 104 & 88 to 118 & $3.220^{* *}$ & 3.00 & IM & $-\mathbf{0 . 1 5 2}$ & $-\mathbf{0 . 0 0 8 0 0}$ \\
D12M6 & 16 & 68 & 56 to 72 & $2.005^{*}$ & 1.82 & IM & 0.0448 & $\mathbf{0 . 1 8 1}$ \\
D15M5 & 8 & 32 & 10 to 54 & $3.344^{* *}$ & 3.02 & IM & $\mathbf{0 . 1 8 0}$ \\
\hline
\end{tabular}


Table 4 Maternal effect QTL. For each QTL the most proximate centromeric marker is listed with the telomeric distance form the marker to the QTL in cM (Dist. M.), the distance from the most centromeric marker to the QTL (Dist. C.), the confidence interval (CI) for the QTL, the LPR associated with the location and the percent of among litter variance that the QTL accounts for. Map identifies the method used to map the QTL (IM = interval mapping and CIM = composite interval mapping). The additive ( $a$ ) and dominance (d) genotypic values are listed with the significance values in bold. LPR values significant at the genome wide level are indicated with a** while * indicates a locus significant at the chromosome level

\begin{tabular}{|c|c|c|c|c|c|c|c|c|}
\hline Marker & Dist. $M$. & Dist. C. & $C I$ & $L P R$ & $\%$ var. & Map & $a$ & $d$ \\
\hline D1Mit7 & 2 & 46 & $38-54$ & $3.547^{* *}$ & 9.53 & IM & -0.138 & 0.324 \\
\hline D6Mit9 & 10 & 60 & $50-68$ & $3.215^{* *}$ & 9.07 & CIM & 0.133 & -0.319 \\
\hline D7Nds1 & 8 & 52 & $44-56$ & $3.390^{* *}$ & 9.13 & IM & -0.170 & -0.268 \\
\hline D13Mit1 & 8 & 8 & 0-18 & $1.898^{*}$ & 5.21 & CIM & -0.117 & 0.251 \\
\hline
\end{tabular}

Table 5 Epistatic interactions between direct effect loci. Two-locus combinations showing significant interaction variance are listed in order of the first chromosome number of the pair. The most proximate centromeric marker to each of the interacting loci is listed (Locus 1 and 2 respectively) along with the telomeric distance of the locus from the maker (Dist. M.) and the distance form the most centromeric marker on the chromosome (Dist. C.). The total LPR score is the likelihood estimate associated with the interaction effect of the loci, which excludes main effects of the two loci but includes all four forms of epistasis. The predominant type(s) of epistasis is listed (Type) along with the genetic value of that form of epistasis (Gen. Val.) and the LPR score of the epistasis component. Significance of the overall LPR scores and of the component scores are indicated with ${ }^{* *}$ for $P<0.05$ and ${ }^{*}$ for $P<0.10$

\begin{tabular}{lcrlcccccc}
\hline Locus 1 & Dist. M. & Dist. C. & Locus 2 & Dist. M. & Dist. C. & Total LPR & Type & Gen val. & Comp. LPR \\
\hline D2Mit1 & 0 & 0 & D14Nds1 & 12 & 12 & 4.344 & AD & 0.399 \\
D3Mit49 & 14 & 82 & D5Mit61 & 20 & 40 & $4.578^{*}$ & AD & -0.501 & $5.774^{* *}$ \\
D3Mit49 & 26 & 94 & D10Mit2 & 34 & 34 & $4.499^{*}$ & DD & -0.353 & 6.1211 \\
D4Mit2 & 16 & 56 & D14Mit7 & 20 & 84 & $4.596^{*}$ & DD & 0.298 & $5.463^{* *}$ \\
D9Mit4 & 14 & 28 & D15Mit5 & 18 & 42 & $4.547^{*}$ & AD & -0.234 & 4.012 \\
D13Mit9 & 36 & 98 & D15Mit2 & 8 & 58 & $5.093^{* *}$ & DD & -0.275 \\
\hline
\end{tabular}

ability level). The interactions were a mixture of $a-x-d$ and $d-x$ - $d$ epistasis.

Most of the significant loci detected in the direct effect epistasis analysis did not map to the same loci as those detected in the single locus analyses. Only two of the four loci involved in the significant interactions are located in a region close to a single locus effect (both involving chromosome 15). However, this does not rule out epistasis between other QTL since the genome-wide significance cutoff is very conservative. Of the 219 pairs of loci identified in the epistasis analysis 43 of the locations involved in these interactions are in the region of one of the single locus QTL. If we view these as protected tests, we would identify considerably more significant epistatic interactions.

A total of 211 pairs of loci show two-locus epistasis for maternal effects on early growth significant at an uncorrected $P<0.05$ level. Twenty-seven of the loci involved in the 211 epistatic maternal effect combinations were within the confidence interval of a single locus maternal effect locus. Ten of the 211 combinations can be considered significant (Table 6). Nine of these combinations show overall epistasis that is significant at the 0.05 level, while one is significant at the 0.10 level. The one locus significant at the 0.10 level shows an $a-x$ - $d$ component of epistasis that is significant at the 0.05 level. Epistatic maternal effect loci show a combination of the four types of epistasis.

There were three chromosome regions that were each involved in more than one epistatic interaction detected in the maternal effect analysis. This suggests that these regions may play a particularly important role in the expression of the maternal effect. Three of the two-locus combinations include a region on chromosome 12, which itself shows no single locus effect (not even a non-significant LPR peak). In addition, two combinations include a region on chromosome 9 , which also shows no single locus effect. Three of the interactions involving chromosome 1 map to a location showing a single locus effect and a fourth maps close to that region.

The interaction between D1Mit74+6 and D6Mit9+16 in the maternal effect analysis is noteworthy because it occurs between a pair of loci that both map within the confidence interval of a single locus effect. Because this pair of loci shows both significant single locus and epistatic effects, it accounts for a large portion of phenotypic variance. A model containing these two loci, and the four epistasis terms accounts for $23.5 \%$ (ie, $R^{2}=0.235$ ) of the among litter variance in growth (with an adjusted $\mathrm{R}^{2}$ of 0.195 or $19.5 \%$ of the variance), where the epistatic terms account for $14.1 \%$ (adjusted $\mathrm{R}^{2}=0.125$ ) while the marginal terms account for the rest. The two-locus combination with the largest LPR score (D1Mit20+10 with D3Mit49+22) is also noteworthy because neither locus shows a significant single locus effect. The complete model with the two loci and the four epistasis terms accounts for a total of $17.9 \%$ of the among litter variance in early growth (adjusted $\mathrm{R}^{2}=0.136$ ). In this case, the epistatic terms account for $15.6 \%$ of the among litter variance, with the single locus terms accounting for the small remaining proportion of variance. The genotypic values of the nine two locus genotypes associated with these two pairs of epistatic interactions are shown in Figure 1. 
Table 6 Epistatic interactions between maternal effect loci. Two-locus combinations showing significant interaction variance are listed in order of the first chromosome number of the pair. The most proximate centromeric marker to each of the interacting loci is listed (Locus 1 and 2 respectively) along with the telomeric distance of the locus from the maker (Dist. M.) and the distance form the most centromeric marker on the chromosome (Dist. C.). The total LPR score is the likelihood estimate associated with the interaction effect of the loci, which excludes main effects of the two loci but includes all four forms of epistasis. The predominant type(s) of epistasis is listed (Type) along with the genetic value of that form of epistasis (Gen. Val.) and the LPR score of the epistasis component. Significance of the overall LPR scores and of the component scores are indicated with ${ }^{* *}$ for $P<0.05$ and $*$ for $P<0.10$

\begin{tabular}{|c|c|c|c|c|c|c|c|c|c|}
\hline Locus 1 & Dist. M. & Dist. C. & Locus 2 & Dist. $M$. & Dist. C. & Total LPR & Type & Gen val. & Comp. LPR \\
\hline D1Mit20 & 10 & 20 & D3Mit49 & 22 & 90 & $5.574^{* *}$ & $\mathrm{AA}$ & 0.530 & 4.672 \\
\hline D1Mit74 & 8 & 40 & D5Mit6 & 2 & 78 & $5.313^{* *}$ & DA & 0.465 & $7.071^{* *}$ \\
\hline D1Mit74 & 6 & 38 & D6Mit9 & 16 & 66 & $4.749^{* *}$ & $\mathrm{DA}$ & 0.402 & $5.578^{* *}$ \\
\hline D1Mit7 & 2 & 46 & D9Mit8 & 36 & 70 & 4.272 & $\mathrm{AD}$ & -0.382 & $5.545^{* *}$ \\
\hline D2Mit1 & 20 & 20 & D11Mit64 & 2 & 48 & $4.892^{* *}$ & DD & 0.567 & 3.098 \\
\hline \multirow[t]{2}{*}{ D4Mit45 } & 6 & 52 & D12Mit6 & 14 & 66 & $5.572^{* *}$ & $\mathrm{AA}$ & -0.533 & 3.770 \\
\hline & & & & & & & $\mathrm{AD}$ & -0.445 & 3.814 \\
\hline D7Mit21 & 26 & 26 & D10Mit2 & 34 & 34 & $5.115^{* *}$ & DD & 0.303 & 3.71 \\
\hline D9Mit4 & 12 & 26 & D12Mit6 & 8 & 58 & $4.800^{* *}$ & $\mathrm{DA}$ & 0.357 & 3.639 \\
\hline D9Mit8 & 2 & 32 & D17Mit16 & 2 & 12 & $5.088^{* *}$ & $\mathrm{AD}$ & -0.407 & 3.889 \\
\hline D10Mit65 & 12 & 66 & D12Mit6 & 8 & 60 & $5.117^{* *}$ & DD & -0.403 & $5.546^{* *}$ \\
\hline
\end{tabular}
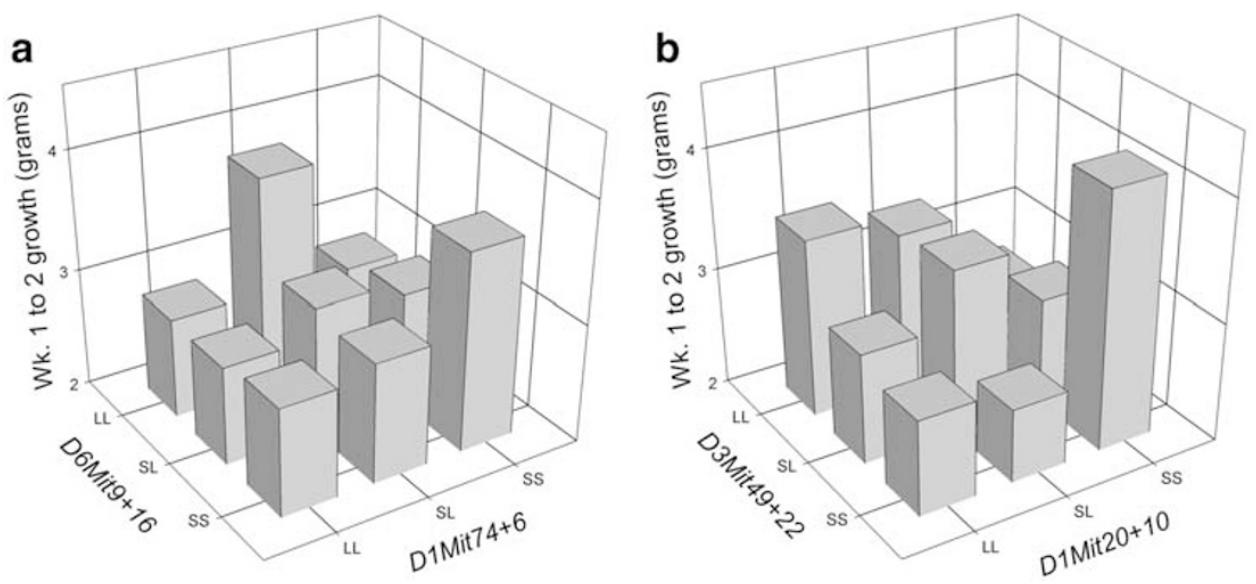

Figure 1 Genotypic values for two-locus combinations showing epistasis for maternal effects on early growth (weight gain from week 1 to 2 in grams). (a) Genotypic values for the D1Mit74+6 by D6Mit9+16 interaction. (b) Genotypic values for the D1Mit20+10 by D3Mit49+22 interaction.

\section{Discussion}

The analysis presented here demonstrates the importance of including both direct and maternal effects in an analysis of QTL contributing to phenotypic variation. If we were to have focused this analysis exclusively on single locus direct genetic effect QTL for early growth we would have identified five loci that together account for $11.8 \%$ of the among individual variance in early growth. In contrast, the analysis presented here, which includes maternal effect loci, identified an additional four loci that together account for $31.5 \%$ of the among litter variance in early growth, which would be expected to account for approximately $23.9 \%$ of the among-individual variance. Epistatic effects on early growth show a similar pattern, with maternal effect loci showing much stronger epistatic effects than the direct effect loci. Clearly, if the goal of this sort of analysis were to understand genetic influences on early growth, then the study focusing exclusively on direct effect loci would be insufficient since it would have missed a major component of genetic architecture in the population.
In addition, since this study examines only postnatal maternal effects it provides no information on the contribution of prenatal effects. The likely influence of prenatal maternal effects on early growth makes the potential contribution of maternal effects to genetic architecture even more important than what is indicated from this analysis. For example, Atchley et al (1991) demonstrated that uterine effects can influence postnatal development but can only be detected when it is possible to cross-foster individuals between uterine environments. Large-scale embryo transfer experiments are prohibitively difficult and as a result, there have been no studies that have examined QTL contributing to prenatal maternal effects.

It is important to keep in mind that the analysis of maternal effect QTL affecting early growth was based on fewer individuals than the analysis of direct effects (158 vs 510), and thus, we might expect a priori, that we would be biased towards detecting a greater contribution of direct effect loci (Beavis, 1994), which was not the case. While this provides fairly strong evidence that maternal effect loci are contributing greater variance than the direct effect loci, when examining the actual effects of 
each locus it is important to keep in mind that, as the sample size in a study decreases, the estimated variance components for each locus are biased upward (Beavis, 1994). As a result, it is not entirely clear whether the maternal effect loci each account for more variance than the direct effect loci, or whether this difference is simply due to bias in estimation. However, the bias in effect size estimation owing to smaller sample size is not expected to inflate the overall proportion of variance explained by the sum of maternal effect loci since the probability of QTL detection is proportionally diminished for each locus. The net result of these two effects is that the total variance explained by the QTL is not generally inflated when sample sizes are smaller (Beavis, 1994). Thus, the fact that we actually found a greater contribution of maternal effect loci further strengthens the assertion that these sorts of loci should be included in genetic analysis of early developmental characters.

Because this analysis was based on an intercross of a single pair of inbred lineages, it is not known whether the patterns of single locus additive and dominance effects provide general rules. However, it is interesting to note that direct effects on early growth were predominantly additive, with little dominance. The additive effects of loci were not in a consistent direction, which is not surprising given that there is very little difference between the SM/J and LG/J lines in early weight gain (Kramer et al, 1998). In contrast, maternal effect loci showed predominantly dominance effects. The dominance effects were a mixture of positive and negative dominance, though more loci showed negative dominance. This fits with the data presented for this cross by Kramer et al (1998), which showed that the growth of the $F_{2}$ individuals was less than the $F_{1}$ or $F_{3}$ individuals, indicating the likelihood of an under-dominant maternal effect on early growth. The difference in genetic architecture of direct and maternal effects implies that these different genetic components of phenotypic variation can contribute to different evolutionary processes. In this population we would expect that direct effects would contribute to directional evolution of growth since they show mostly additive effects while maternal effects would play less of a role in directional evolution since they show little additive effect. In contrast, maternal effects would be expected to play more of a role in evolutionary processes that rely on the presence of dominance such as maintenance of genetic variation and inbreeding or outbreeding depression.

This analysis also illustrates the importance of considering epistatic interactions in a QTL analyses. Most loci that appear to show strong epistatic interactions do not show significant single locus effects. As a result, if we had limited our analysis of epistasis to interactions between loci showing significant single locus effects we would have missed most of these interacting pairs. Considerably more pairs show epistasis for maternal effects than for direct effects, suggesting that epistasis is a particularly important component of maternal effect genetic variation. This is especially striking given that the maternal effect analysis is based on so many fewer individuals. These results, in combination with the single locus data, also suggest that non-additive genetic effects are predominant over additive effects in the genetic architecture of maternal effects. We also see that a number of the loci involved in epistatic interactions for maternal effects are involved in more than one interaction, suggesting that some loci play a central role in the expression of maternal effects by modulating the effects of other loci. There are also combinations of loci showing epistasis for maternal effects that each show significant single locus effects. As a result, these combinations show very large influences on the phenotype. For example, the interaction between D1Mit74+6 and D6Mit9+16 (see Figure 1), accounts for $23.5 \%$ of the among litter variance in growth when we fit a model containing additive, dominance and epistatic effects of the two loci.

The occurrence of a small genetic covariance between the direct and maternal effects suggests that we might expect to find some shared QTL locations. However, we find no evidence for pleiotropy since no maternal effect QTL maps to the same chromosome as a direct effect QTL. This is not to say that there are no shared loci, it simply means that we did not detect any in this analysis. As with all QTL analyses, the probability that we would detect the pleiotropic effects of a locus is less than the probability that we would detect the effect of a locus on a single trait. This is due to the fact that the probability of co-detection (ie, detecting an affect of a locus on two different traits) depends on us detecting the effect of a locus on two different, somewhat independent characters, and thus the probability of co-detection it is likely to be much smaller than the probabilities of detection associated with each character. As a result, we have less power to detect pleiotropy than we do to detect single locus effects.

Because the maternal effect is defined here as the influence of the nurse on the growth of her foster pups (ie, it is a trait measured in the fostered individuals, not a direct characteristic of the nurse-dam) it is a composite phenotype that cannot be directly attributed to any single maternal character. This character is referred to as 'maternal performance' in quantitative genetics (see Wolf et al, 1998), and is usually what is estimated as the maternal effect when components of phenotypic variation are partitioned statistically. Given the biology of the mouse, we would expect maternal performance to be attributable primarily to maternal lactation quality, quality of the nest environment provided by the female and other behaviors associated with maternal care such as grooming (eg, Caldji et al, 2000; see Peripato and Cheverud, in press). Lactation quality could include factors such as quantity of milk produced, nutritional quality (such as caloric content, and relative content of fats, carbohydrates and proteins in milk) and non-nutrient components such as hormones. Nest quality could include characteristics of nest construction such as use of insulation materials, depth of nest, but may also include factors such as the quality of the thermal environment that the mother actively provides by tending the nest (eg, Bult and Lynch, 2000). These characters provide a starting point in the identification of genes that might underlie the maternal effect QTL.

While we do not have data on any of these maternal characteristics, there are a number of phenotypes that have been measured on the nurse dams and can be used to examine whether any particular maternal characteristics appear to covary with pup growth. We find that reproductive fatpad mass at necropsy is positively correlated with pup growth $\left(r^{2}=0.17, P=0.047\right)$, as is total body weight at necropsy $\left(r^{2}=0.24, P=0.0022\right)$ and tail- 
length $\left(r^{2}=0.18, P=0.020\right)$. These correlations suggest some physiological relationship between expression of some maternal characters and maternal effects. Because the individuals used in this analysis have also been used in mapping studies of body size, and adiposity (Vaughn et al, 1999; Cheverud et al 2001) we can examine whether the maternal effect QTL map to the same genomic locations as loci influencing these other characters. We find that one maternal effect QTL (D6Mit9+10) maps close to a locus influencing adiposity (Cheverud et al, 2001) and shows the same pattern of a positive additive effect and negative dominance effect, suggesting a single locus that influences both characters through a similar mode. Three loci mapped near loci known to influence female body size, as measured by body weight at necropsy $(D 7 N d s 1+8)$, tail length $(D 1 M i t 7+2)$ or both traits (D6Mit9+10). The remaining maternal effect locus $(D 13 M i t 1+8)$ does not appear to be associated with loci that have been mapped for any of the adult characters that correlate with fostered pup growth. All loci also map close to at least one age specific weight or growth location identified by Vaughn et al (1999) for this intercross, though none of these other characters correlate with fostered pup growth. The largest maternal effect locus $(D 7 N d s 1+8)$ falls at the same chromosomal location as a locus influencing all post-weaning weekly weights (week 4 through 10) and growth during all intervals measured (but is not correlated with week 1 to 2 week growth as estimated here). Thus, there may be a locus in that region that affects some fundamental physiological character that results in pleiotropic effects on the expression of a large number of traits.

Since groups of mice develop together in litters, there is also the possibility that effects analogous to maternal effects could occur when pups influence each other's phenotypes. These sorts of 'sib effects' would add an additional mode through which the genotype of one individual might influence the phenotype of another individual (ie, indirect genetic effects can occur). These effects could potentially appear as maternal genetic effects, since the maternal and offspring genotypes are correlated due to relatedness. However, we essentially can rule out the possibility that the maternal effect loci detected here are due to sib effects since the maternal effect loci all show dominance and there is no correlation between the dominance values of loci in mothers and their progeny (ie, dominance is not heritable). Furthermore, a post hoc analysis, substituting the genotypes of the nurse-dams' mates for their genotypes, strongly supports the conclusion that these are indeed maternal effect loci because, as expected, the foster pups' phenotypes do not map to the genotypes of these males (who had no contact with any of the pups but are just as related to the non-fostered pups as the nurse-dam).

In order to understand the functional basis of these maternal effects, and to begin to identify the sorts of loci involved, more research will be required. However, we have clearly demonstrated the value in including maternal effects in an analysis of genetic architecture. Our results imply that a thorough understanding of genetic architecture underlying many characters, especially those expressed early in life, will benefit greatly from an approach that is not focused exclusively on direct effects of genes.

\section{Acknowledgements}

We thank RA Brito, S Cropp, T Ehrlich, PX Kover and AC Peripato for frequent valued input during the development of this work and L Leamy for generously sharing the SAS programs used in the analysis presented here. This work was supported by a National Science Foundation (USA) Postdoctoral Fellowship in Biological Informatics to JBW and by grants from the National Institutes of Health (DK52514) and the National Science Foundation (DEB-9726433) to JMC.

\section{References}

Atchley WR, Logsdon TE, Cowley DE, Eisen EJ (1991). Uterine effects, epigenetics and postnatal skeletal development in the mouse. Evolution 45: 891-909.

Beavis WD (1994). The power and deceit of QTL experiments: lessons from comparative QTL studies. In: 49th Annual Corn and Sorghum Research Conference, American Seed Trade Association, Washington, DC. pp 252-268.

Blackith RE, Reyment RA (1971). Multivariate Morphometrics. Academic Press: NY.

Bult A, Lynch CB (2000). Breaking through artificial selection limits of an adaptive behavior in mice and the consequences for correlted responses. Behav Genet 30: 193-206.

Caldji C, Diorio J, Meaney MJ (2000). Variations in maternal care in infancy regulate the development of stress reactivity. Biol Psych 48: 1164-1174.

Chai C (1956). Analysis of quantitative inheritance of body size in mice. I. Hybridization and maternal influence. Genetics 41: 157-164.

Cheverud JM (2000). Detecting epistasis among quantitative trait loci. In: Wolf JB, Brodie ED, III, Wade MJ (eds) Epistasis and the Evolutionary Process, Oxford University Press: NY. pp 58-81.

Cheverud JM (2001). A simple correction for multiple comparisons in interval mapping genome scans. Heredity 87: 52-58.

Cheverud JM, Moore AJ (1994). Quantitative genetics and the role of the environment provided by relatives in the evolution of behavior, In: Boake CRB (ed) Quantitative Genetic Studies of Behavioral Evolution, University of Chicago Press: Chicago. pp 67-100

Cheverud JM, Routman EJ (1995). Epistasis and its contribution to genetic variance components. Genetics 139: 1455-1461.

Cheverud JM, Routman EJ, Duarte FM, Van Swinderen B, Cothran K, Perel C (1996). Quantitative trait loci for murine growth. Genetics 142: 1305-1319.

Cheverud JM, Vaughn TT, Pletcher LS, Peripato AC, Adams ES, Erikson CF et al (2001). Genetic architecture of adiposity in the cross of LG/J and SM/J inbred mice. Mamm Genome 12: 3-12.

Churchill GA, Doerge RW (1994). Empirical threshold values for quantitative trait mapping. Genetics 138: 963-971.

Corva PM, Medrano JF (2001). Quantitative trait loci (QTLs) mapping for growth traits in the mouse: A review. Genet Select Evol 33: 105-132.

Cowley DE, Pomp D, Atchley WR, Eisen EJ, Hawkins-Brown D (1989). The impact of maternal uterine genotype on postnatal growth and adult body size in mice. Genetics 122: 193-203.

Falconer DS (1965). Maternal effects and selection response. In: Geerts SJ (ed) Genetics Today: Proceedings of 11th International Congress of Genetics, Pergamon Press: Oxford. pp 763-74.

Goodale H (1938). A study of the inheritance of body weight in the albino mouse by selection. J Hered 29: 101-112.

Haley CS, Knott SA (1992). A simple regression method for mapping quantitative trait loci in line crosses using flanking markers. Heredity 69: 315-324.

Kirkpatrick M, Lande R (1989). The evolution of maternal characters. Evolution 43: 485-503.

Kramer MG, Vaughn TT, Pletscher LS, King-Ellison K, Adams 
E, Erikson C et al (1998). Genetic variation for body weight gain and composition in the intercross of Large (LG/J) and Small (SM/J) inbred strains of mice. Genet Mol Biol 21: 211218.

Lander ES, Botstein D (1989). Mapping Mendelian factors underlying quantitative traits using RFLP linkage maps. Genetics 121: 185-199.

Leamy L, Routman E, Cheverud JM (1999). Quantitative trait loci for early and late developing skull characters in mice: a test of the genetic independence model of morphological integration. Am Nat 153: 201-214.

Legates JE (1972). The role of maternal effects in animal breeding: IV. Maternal effects in laboratory species. J Anim Sci 35: 1294-1302.

Lynch M, Walsh B (1998). Genetics and Analysis of Quantitative Traits. Sinauer Assoc: Sunderland, MA.

Macarthur J (1944). Genetics of body size and related characters. I. Selection of small and large races of the laboratory mouse. Am Nat 78: 142-157.

Mousseau TA, Dingle H (1991). Maternal effects in insect life histories. Ann Rev Ent 36: 511-534.

Mousseau TA, Fox C (1998a). Maternal Effects as Adaptations. Oxford University Press: NY.

Mousseau TA, Fox C (1998b). The adaptive significance of maternal effects: moms do more than make babies. Trends Ecol Evol 13: 403-406.

Newman S, Rutledge JJ, Riska B (1989). Estimation of prenatal maternal genetic effects. J Anim Breed Gen 106: 30-38.

Oksh HA, Sutherland TM, Williams JS (1967). Prenatal and postnatal maternal influence on growth in mice. Genetics 57: 79-94.

Peripato AC, Cheverud JM. Genetic influences on maternal care. Amer Nat (in press).
Reifsnyder PC, Churchill G, Leiter EH (2000). Maternal environmental and genotype interact to establish diabesity in mice. Genome Res 10: 1568-1578.

Reznick DN (1991). Maternal effects in fish life histories. In: Dudley EC (ed) The Unity of Evolutionary Biology: Proceedings of the Fourth International Congress of Systematic and Evolutionary Biology, vol. II., Dioscorides Press: Portland. pp 780-793.

Riska BJ, Rutledge JJ, Atchley WR (1985). Covariances between direct and maternal genetic effects in mice, with a model of persistent environmental influences. Genet Res 42: 287-297.

Roach DA, Wulff RD (1987). Maternal effects in plants. Ann Rev Ecol Syst 18: 209-235.

Routman EJ, Cheverud JM (1997). Gene effects on a quantitative trait: two-locus epistatic effects measured at microsatellite markers and at estimated QTL. Evolution 51: 1654-1662.

SAS $^{\circledR}$ Institute Inc (1992). SAS ${ }^{\circledR}$ Technical Report P-229, SAS/STAT ${ }^{\circledR}$ Software: Changes and Enhancements, Release 6.07. SAS Institute Inc., Cary, N.C.

Vaughn TT, Pletscher LS, Peripato A, King-Ellison K, Adams E, Erikson C et al (1999). Mapping quantitative trait loci for murine growth - a closer look at genetic architecture. Genet Res 74: 313-322.

Wade MJ (2001). Maternal effect genes and the evolution of sociality in haplo-diploid organisms. Evolution 55: 453-458.

Wolf JB (2000). Gene interactions from maternal effects. Evolution, 54: 1882-1898.

Wolf JB, Brodie ED III, Cheverud JM, Moore AJ, Wade MJ (1998). Evolutionary consequences of indirect genetic effects. Trends Ecol Evol 13: 64-69.

Zeng Z-B (1993). Theoretical basis for separation of multiple linked gene effects in mapping quantitative trait loci. Proc Nat Acad Sci USA 90: 10972-10976. 